УДК 519.21

\title{
Numerical Investigation of Solutions to a Reaction-diffusion System with Variable Density
}

\author{
Shahlo A. Sadullaeva* \\ Tashkent University of Information Technology \\ Amir Temur, 108, Tashkent, 700084, \\ Uzbekistan
}

Received 15.10.2015, received in revised form 06.11.2014, accepted 30.12.2014

In this paper we demonstrate the possibilities of the self-similar and approximately self-similar approaches for studying solutions of a nonlinear mutual reaction-diffusion system. The asymptotic behavior of compactly supported solutions and free boundary is studied. Based on established qualitative properties of solutions numerical computation is carried out. The solutions are presented in visualization form, which allows observing evolution of the studied process in time.

Keywords: double nonlinear reaction-diffusion system, self-similar solutions, asymptotics, numerical calculations.

DOI: 10.17516/1997-1397-2016-9-1-90-101.

\section{Introduction}

In the domain $Q=\left\{(t, x): t>0, x \in \mathbb{R}^{N}\right\}$ properties are investigated of the process of a nonlinear diffusion-reaction with variable density described by the following system:

$$
\begin{gathered}
\frac{\partial(\rho(x) u)}{\partial t}=\operatorname{div}\left(|x|^{n} v^{m_{1}-1}|\nabla u|^{p-2} \nabla u\right)+\rho(x) \gamma(t) u^{\beta_{1}} \\
\frac{\partial(\rho(x) v)}{\partial t}=\operatorname{div}\left(|x|^{n} u^{m_{2}-1}|\nabla v|^{p-2} \nabla v\right)+\rho(x) \gamma(t) v^{\beta_{2}} \\
u(0, x)=u_{0}(x) \geqslant 0, \\
v(0, x)=v_{0}(x) \geqslant 0, x \in \mathbb{R}^{N},
\end{gathered}
$$

where $m_{1}, m_{2}, n \in \mathbb{R}, \beta_{1}, \beta_{2} \geqslant 1, p \geqslant 2$ are given positive numbers.

$$
\begin{aligned}
& \nabla(\cdot)-\operatorname{grad}(\cdot), \quad u_{0}(x), v_{0}(x) \geqslant 0, \quad x \in \mathbb{R}^{N}, \\
& \rho(x)=|x|^{-l}, \quad l>0, \quad 0<\gamma(\mathrm{t}) \in C(0, \infty) .
\end{aligned}
$$

System (1) describes different physical processes in two componential inhomogeneous nonlinear media, for example, the processes of mutual reaction-diffusions, heat conductivity, the theory of combustion, the theory of a polytrophic filtration of a liquid and a gas in the presence of a source whose power is equal to $\rho(x) \gamma(t) u^{\beta_{1}}, \rho(x) \gamma(t) v^{\beta_{2}}$. In works [1-6] were considered particular cases of the system (1), when $\gamma(\mathrm{t})=1, n=l=0, p=2$ and we investigated arising different types of solutions depending on the parameters of system (1).

System (1) is degenerate in the domain, where $u=v=0$ and may have no classical solutions. Therefore, the weak solutions of system (1) are studied, having physical sense. Namely functions $0 \leqslant u, v \in C(Q)$ such that $|x|^{n} v^{m_{1}-1}|\nabla u|^{p-2} \nabla u,|x|^{n} u^{m_{2}-1}|\nabla v|^{p-2} \nabla v \in C(Q)$ satisfying the

*orif_sh@list.ru

(C) Siberian Federal University. All rights reserved 
integral identity in the sense of distributions [1]. For solutions of system (1) may take place the phenomena of finite velocity of propagation and space localization of disturbance, i.e. there may exist functions $l_{1}(t), l_{2}(t)$, such that $u(t, x) \equiv 0$ and $v(t, x) \equiv 0$ if $|x| \geqslant l_{1}(t)$ and $|x| \geqslant l_{2}(t)$. In the case $l_{1}(t), l_{2}(t)<\infty$, for $t>0$ a solution to problem (1)-(2) is called a space localization of disturbance. The surfaces $|x|=l_{1}(t)$ and $|x|=l_{2}(t)$ are called a free boundary or a front.

In the present work we suggest a method of construction of self-similar equations system (1) based of splitting of system (1), and study asymptotics of compactly support solutions and a free boundary and asymptotics of self-similar solutions for the quick diffusion case. It is shown that the coefficient of the main member of the asymptotics of the solution satisfies a certain system of nonlinear algebraic system equation. Based on established qualitative properties of the solution, using approximately self-similar solutions, numerical experiments, visualization of processes described by reaction-diffusion system (1) with variable density were carried out.

\section{Construction of a self-similar system of equations}

Studying different properties of solutions to system (1) is a complicated problem, even for particular cases of system (1) [2,6-9]. In these works for particular cases of system (1)-(2) the effectiveness was shown of the self-similar approach for studying different properties of solutions to problem (1)-(2). Below a new method (nonlinear splitting) of construction of a self-similar and an approximately self-similar system is suggested. This method gives us a more simple way of investigation of qualitative properties of solutions to problem (1)-(2).

For construction of a self-similar and an approximately self-similar system for system (1) solutions $u(t, x), v(t, x)$ to system of equations (1) are searched in the form

$$
\begin{aligned}
& u(t, x)=\bar{u}(t) w(\tau(t), \varphi(|x|)), \\
& v(t, x)=\bar{v}(t) z(\tau(t), \varphi(|x|)),
\end{aligned}
$$

where

$$
\bar{u}(t)=\left[T+\int_{0}^{t} \gamma(t) d t\right]^{-\frac{1}{\beta_{1}-1}}, \quad \bar{v}(t)=\left[T+\int_{0}^{t} \gamma(t) d t\right]^{-\frac{1}{\beta_{2}-1}}, \quad T>0,
$$

and functions $\tau, \varphi$ will be choosen below.

Substituting (3) in system (1) reduces it to the following system of equations

$$
\begin{aligned}
\frac{\partial w}{\partial \tau} & =\varphi^{1-s} \frac{\partial}{\partial \varphi}\left(\varphi^{s-1} z^{m_{1}-1}\left|\frac{\partial w}{\partial \varphi}\right|^{p-2} \frac{\partial w}{\partial \varphi}\right)+\gamma(t) \bar{u}^{\beta_{1}-(p-1)} \bar{v}^{-\left(m_{1}-1\right)}\left(w+w^{\beta_{1}}\right), \\
\frac{\partial z}{\partial \tau} & =\varphi^{1-s} \frac{\partial}{\partial \varphi}\left(\varphi^{s-1} w^{m_{2}-1}\left|\frac{\partial z}{\partial \varphi}\right|^{p-2} \frac{\partial z}{\partial \varphi}\right)+\gamma(t) \bar{u}^{-\left(m_{2}-1\right)} \bar{v}^{\beta_{2}-(p-1)}\left(z+z^{\beta_{2}}\right),
\end{aligned}
$$

where the functions $\tau, \varphi$ are chosen as

$$
\begin{gathered}
\tau(t)=\int_{0}^{t} \bar{v}^{m_{1}-1}(\eta) \bar{u}^{p-2}(\eta) d \eta=\int_{0}^{t} \bar{u}^{m_{2}-1}(\eta) \bar{v}^{p-2}(\eta) d \eta \\
\varphi(r)=\frac{1}{p_{1}}|r|^{p_{1}}, \quad|r|=\sqrt{\sum_{i=1}^{N} x_{i}^{2}}, \quad p_{1}=\frac{p-(n+l)}{p}, \quad s=p \frac{N-l}{p-(n+l)}, l+n<p .
\end{gathered}
$$

It is easy to establish that system (4) has approximately self-similar solutions of the form

$$
\begin{aligned}
& w(\tau, \varphi)=f(\xi), \\
& z(\tau, \varphi)=\psi(\xi),
\end{aligned}
$$


where $\xi=\frac{\varphi(x)}{\tau^{1 / p}}$ and the functions $f, \psi$ satisfy to the following approximately self-similar system of equations

$$
\begin{gathered}
\xi^{1-s} \frac{d}{d \xi}\left(\xi^{s-1} \psi^{m_{1}-1}\left|\frac{d f}{d \xi}\right|^{p-2} \frac{d f}{d \xi}\right)+\frac{\xi}{p} \frac{d f}{d \xi}+\gamma(t) \tau(t) \bar{u}^{\beta_{1}-(p-1)} \bar{v}^{-\left(m_{1}-1\right)}\left(f+f^{\beta_{1}}\right)=0, \\
\xi^{1-s} \frac{d}{d \xi}\left(\xi^{s-1} f^{m_{2}-1}\left|\frac{d \psi}{d \xi}\right|^{p-2} \frac{d \psi}{d \xi}\right)+\frac{\xi}{p} \frac{d \psi}{d \xi}+\gamma(t) \tau(t) \bar{u}^{-\left(m_{2}-1\right)} \bar{v}^{\beta_{2}-(p-1)}\left(\psi+\psi^{\beta_{2}}\right)=0,
\end{gathered}
$$

It is easy to provej that

$$
\begin{aligned}
& \gamma(t) \bar{u}^{\beta_{1}-(p-1)} \bar{v}^{-\left(m_{1}-1\right)} \tau(t) \rightarrow \mathrm{const}, \text { as } t \rightarrow \infty, \\
& \gamma(t) \bar{u}^{-\left(m_{1}-1\right)} \bar{v}^{\beta_{2}-(p-1)} \tau(t) \rightarrow \mathrm{const}, \text { as } t \rightarrow \infty
\end{aligned}
$$

if $0<\gamma(t) \in H$, where $H$ is Hardy's body [2]. In this case system (1) becomes self-similar. Therefore it is possible to say that (7) is an asymptotically self-similar system for system of equations (1).

Let $\gamma(t)=$ const. Then approximately self-similar system (7) has a self-similar form if

$$
\left(\beta_{2}-1\right)\left(p-\left(m_{1}+1\right)\right)=\left(\beta_{1}-1\right)\left(p-\left(m_{2}+1\right)\right)
$$

In this case for the functions $f, \psi$ we have the following self-similar system

$$
\begin{gathered}
\xi^{1-s} \frac{d}{d \xi}\left(\xi^{s-1} \psi^{m_{1}-1}\left|\frac{d f}{d \xi}\right|^{p-2} \frac{d f}{d \xi}\right)+\frac{\xi}{p} \frac{d f}{d \xi}+a_{1}\left(f+f^{\beta_{1}}\right)=0 \\
\xi^{1-s} \frac{d}{d \xi}\left(\xi^{s-1} f^{m_{2}-1}\left|\frac{d \psi}{d \xi}\right|^{p-2} \frac{d \psi}{d \xi}\right)+\frac{\xi}{p} \frac{d \psi}{d \xi}+a_{2}\left(\psi+\psi^{\beta_{2}}\right)=0
\end{gathered}
$$

where

$$
\begin{aligned}
& a_{1}=\left(\beta_{1}-1\right)\left(\beta_{2}-1\right) /\left[\left(\beta_{1}-1\right)\left(\beta_{2}-1\right)-\left(\left(m_{1}-1\right)\left(\beta_{1}-1\right)+(p-2)\left(\beta_{2}-1\right)\right)\right], \\
& a_{2}=\left(\beta_{1}-1\right)\left(\beta_{2}-1\right) /\left[\left(\beta_{1}-1\right)\left(\beta_{2}-1\right)-\left(\left(m_{2}-1\right)\left(\beta_{2}-1\right)+(p-2)\left(\beta_{1}-1\right)\right)\right] .
\end{aligned}
$$

We notice that in the singular case $\beta_{1}=\left(m_{1}+p-2\right), \beta_{2}=\left(m_{2}+p-2\right)$ positive solutions system (10) in the case of one equation were studied in [9].

\section{Asymptotics of self-similar solutions}

Now we will study asymptotics of weak compactly supported solutions (c.s.) to system (10) when $\gamma(t)=$ const.

Consider system of equations (10) with the following boundary condition

$$
\begin{aligned}
& f(0)=c_{1}>0, \quad f(b)=0 \\
& \psi(0)=c_{2}>0, \quad \psi(b)=0
\end{aligned}
$$

where $0<b<+\infty$.

The existence of a self-similar weak solution to problem (10)-(11) for one equation, in the case $\gamma(t)=0, n=l=0, \quad p=2$ was studied in [1] and conditions for the existence of c.s. solutions were obtained. Asymptotic behavior of a self similar c.s. solution ere established for the case 
one equation in [10] and for other system, but with other nonlinear coefficients in [3-6,11] when $\mathrm{p}=2$.

Now we will study the asymptotic of a c.s. solution system (10) in the case $p+m_{1}-3>0$, $p+m_{2}-3>0$. For this goal we will transform system (10) to the convenient form for investigation by using the following transformations

$$
\begin{aligned}
& f(\xi)=\bar{f}(\xi) y_{1}(\eta), \quad \eta=-\ln \left(a-\xi^{\lambda}\right), \quad 0<\xi<a^{1 / \lambda}, \\
& \psi(\xi)=\bar{\psi}(\xi) y_{2}(\eta),
\end{aligned}
$$

where

$$
\begin{gathered}
\bar{f}(\xi)=\left(a-\xi^{\lambda}\right)_{+}^{q_{1}}, \quad \bar{\psi}(\xi)=\left(a-\xi^{\lambda}\right)_{+}^{q_{2}}, \quad \lambda=\frac{p}{p-1}, \\
q_{1}=\frac{(p-1)\left(p-\left(m_{1}+1\right)\right)}{q}, \quad q_{2}=\frac{(p-1)\left(p-\left(m_{2}+1\right)\right)}{q}, \\
p>m_{1}+1, \quad p>m_{2}+1, \quad a>0, \quad q=(p-2)^{2}-\left(m_{1}-1\right)\left(m_{2}-1\right) .
\end{gathered}
$$

Case $(p-2)^{2}-\left(m_{1}-1\right)\left(m_{2}-1\right)=0$ is a singular case. In this case the asymptotic of a solutions of the system (10) have another behavior. This case is required an additional investigation.

Theorem 1. Let $q_{1}>0, q_{2}>0$. Then c.s. solutions to system (10) as $\eta \rightarrow+\infty\left(\xi \rightarrow a^{1-\frac{1}{p}}\right)$ have the asymptotic

$$
\begin{aligned}
& f(\xi)=y_{1}^{0} \bar{f}(\xi)(1+o(1)), \\
& \psi(\xi)=y_{2}^{0} \bar{\psi}(\xi)(1+o(1)),
\end{aligned}
$$

where $0<y_{i}^{0}<+\infty(i=1,2)$, if one of following conditions is satisfied:

1) $\beta_{i}>\frac{q_{i}-1}{q_{i}}, i=1,2$, then $\left(y_{1}^{0}, y_{2}^{0}\right)$ are the real roots of the following system of nonlinear algebraic equations

$$
\begin{gathered}
\left(y_{2}^{0}\right)^{m_{1}-1}\left(y_{1}^{0}\right)^{p-2}=c_{1}, \\
\left(y_{1}^{0}\right)^{m_{2}-1}\left(y_{2}^{0}\right)^{p-2}=c_{2}, \\
c_{i}=\frac{1}{p\left(\lambda q_{i}\right)^{p-1}}, \quad i=1,2,
\end{gathered}
$$

i.e.

$$
\begin{gathered}
y_{1}^{0}=\left(\frac{\lambda^{p-2} q_{2}^{p-1} p}{q_{1}\left(\lambda p q_{1}\right)^{\frac{1}{p-2}}}\right)^{\frac{m_{1}-2}{q}}\left[\frac{1}{\lambda p q_{1}}\right]^{\frac{1}{p-2}} \frac{1}{\lambda q_{1}}, \\
y_{2}^{0}=\left(\frac{q_{1}\left(\lambda p q_{1}\right)^{\frac{1}{p-2}}}{\lambda^{p-2} q_{2}^{p-1} p}\right)^{\frac{p-2}{q}},
\end{gathered}
$$

where $q_{i}, i=1,2$ are the numbers defined above;

2) $\beta_{i}=\frac{q_{i}-1}{q_{i}}, i=1,2$, then $\left(y_{1}^{0}, y_{2}^{0}\right)$ are the real roots of the the following system of nonlinear algebraic equations

$$
\begin{aligned}
& q_{1}^{p-1}\left(y_{2}^{0}\right)^{m_{1}-1}\left(y_{1}^{0}\right)^{p-2}+\frac{a_{1}\left(y_{1}^{0}\right)^{\beta_{1}-1}}{a \lambda^{p} q_{1}}=\frac{1}{p \lambda^{p-1}}, \\
& q_{2}^{p-1}\left(y_{1}^{0}\right)^{m_{2}-1}\left(y_{2}^{0}\right)^{p-2}+\frac{a_{2}\left(y_{2}^{0}\right)^{\beta_{2}-1}}{a \lambda^{p} q_{2}}=\frac{1}{p \lambda^{p-1}} .
\end{aligned}
$$


Proof. In order to prove Theorem 1 we use transformation (12). Transformations (12) reduces self-similar system (10) to the following form

$$
\begin{aligned}
& \frac{d}{d \eta} L_{1}\left(y_{1}, y_{2}\right)+\left(\frac{s}{\lambda} \phi(\eta)-q_{1}\right) L_{1}\left(y_{1}, y_{2}\right)+\frac{y_{1}^{\prime}-q_{1} y_{1}}{\lambda^{p-1}} \phi(\eta)+\frac{a_{1}}{\lambda^{p}} \phi(\eta)\left(y_{1}+\phi_{1}(\eta) y_{1}^{\beta_{1}}\right)=0 \\
& \frac{d}{d \eta} L_{1}\left(y_{1}, y_{2}\right)+\left(\frac{s}{\lambda} \phi(\eta)-q_{2}\right) L_{1}\left(y_{1}, y_{2}\right)+\frac{y_{2}^{\prime}-q_{2} y_{2}}{\lambda^{p-1}} \phi(\eta)+\frac{a_{2}}{\lambda^{p}} \phi(\eta)\left(y_{2}+\phi_{2}(\eta) y_{2}^{\beta_{2}}\right)=0
\end{aligned}
$$

where

$$
\begin{aligned}
& \phi(\eta)=\frac{e^{-\eta}}{a-e^{-\eta}}, \quad \phi_{i}(\eta)=e^{-\eta q_{i}\left(\beta_{i}-1\right)}, \quad i=1,2 \\
& L_{1}\left(y_{1}, y_{2}\right)=y_{2}^{m_{1}-1}\left(\left|y_{1}^{\prime}-q_{1} y_{1}\right|\right)^{p-2}\left(y_{1}^{\prime}-q_{1} y_{1}\right) \\
& L_{2}\left(y_{1}, y_{2}\right)=y_{1}^{m_{2}-1}\left(\left|y_{2}^{\prime}-q_{2} y_{2}\right|\right)^{p-2}\left(y_{2}^{\prime}-q_{2} y_{2}\right)
\end{aligned}
$$

and $\lambda, q_{i}, a_{i}, i=1,2$ are the numbers defined above.

Such transformation (12) allows us to reduce studying of the asymptotics of solutions to system (10) as $\eta \rightarrow \infty$ to studying those solutions of system (16), which in some neighborhood of $+\infty$ satisfy the inequalities

$$
\frac{d y_{i}}{d \eta}-q_{i} y_{i} \neq 0, \quad y_{i}(\eta)>0, \quad i=1,2
$$

First we show that solutions $y_{1}(\eta), y_{2}(\eta)$ to system (16) have finite limits as $\eta \rightarrow \infty$.

We introduce the notation

$$
v_{i}(\eta)=L_{i}\left(y_{1}, y_{2}\right), \quad i=1,2
$$

Then system (16) may be rewritten in the form

$$
\begin{aligned}
& v_{1}^{\prime}=-\left(\frac{s}{\lambda} \phi(\eta)-q_{1}\right) v_{1}-\frac{y_{1}^{\prime}-q_{1} y_{1}}{\lambda^{p-1}} \phi(\eta)-\frac{a_{1}}{\lambda^{p}} \phi(\eta)\left(y_{1}+\phi_{1}(\eta) y_{1}^{\beta_{1}}\right), \\
& v_{2}^{\prime}=-\left(\frac{s}{\lambda} \phi(\eta)-q_{2}\right) v_{2}-\frac{y_{2}^{\prime}-q_{2} y_{2}}{\lambda^{p-1}} \phi(\eta)-\frac{a_{2}}{\lambda^{p}} \phi(\eta)\left(y_{2}+\phi_{2}(\eta) y_{2}^{\beta_{2}}\right) .
\end{aligned}
$$

To analyze the solutions to this system, we introduce the auxiliary functions

$$
\begin{aligned}
& \theta_{1}\left(\mu_{1}, \eta\right)=-\left(\frac{s}{\lambda} \phi(\eta)-q_{1}\right) \mu_{1}-\frac{y_{1}^{\prime}-q_{1} y_{1}}{\lambda^{p-1}} \phi(\eta)-\frac{a_{1}}{\lambda^{p}} \phi(\eta)\left(y_{1}+\phi_{1}(\eta) y_{1}^{\beta_{1}}\right) \\
& \theta_{2}\left(\mu_{2}, \eta\right)=-\left(\frac{s}{\lambda} \phi(\eta)-q_{2}\right) \mu_{2}-\frac{y_{2}^{\prime}-q_{2} y_{2}}{\lambda^{p-1}} \phi(\eta)-\frac{a_{2}}{\lambda^{p}} \phi(\eta)\left(y_{2}+\phi_{2}(\eta) y_{2}^{\beta_{2}}\right)
\end{aligned}
$$

where $\mu_{i}, i=1,2$, are real numbers. For each value of $\mu_{i}$ the function $\theta_{i}\left(\mu_{i}, \eta\right)$ preserves the sign on some interval $\left[\eta_{\mu_{i}},+\infty\right) \subset\left[\eta_{0},+\infty\right)\left(0<\eta_{0}<\eta_{\mu_{i}}\right)$ and for all $\eta \in\left[\eta_{\mu_{i}},+\infty\right)$ takes place one of the inequalities

$$
\theta_{1}\left(\mu_{i}, \eta\right)>0, \quad \theta_{1}\left(\mu_{i}, \eta\right)<0 .
$$

Given the theorem of Bohl [13] for the functions $v_{i}(\eta)$ there are a limit in $\eta \in\left(\eta_{\mu_{i}},+\infty\right)$ :

$$
\lim _{\eta \rightarrow+\infty} v_{i}(\eta)<+\infty, \quad \lim _{\eta \rightarrow+\infty} v_{i}^{\prime}(\eta)=0 .
$$

Therefore

$$
\lim _{\eta \rightarrow+\infty} y_{i}(\eta)=y_{i}^{0}<+\infty, \quad \lim _{\eta \rightarrow+\infty} y_{i}^{\prime}(\eta)=0
$$


Then we have

$$
\begin{aligned}
& \lim _{\eta \rightarrow+\infty} v_{1}^{\prime}=\lim _{\eta \rightarrow+\infty}\left\{-\left(\frac{s}{\lambda} \phi(\eta)-q_{1}\right) \mu_{1}-\frac{y_{1}^{\prime}-q_{1} y_{1}}{\lambda^{p-1}} \phi(\eta)-\frac{a_{1}}{\lambda^{p}} \phi(\eta)\left(y_{1}+\phi_{1}(\eta) y_{1}^{\beta_{1}}\right)\right\}=0, \\
& \lim _{\eta \rightarrow+\infty} v_{2}^{\prime}=\lim _{\eta \rightarrow+\infty}\left\{-\left(\frac{s}{\lambda} \phi(\eta)-q_{2}\right) \mu_{2}-\frac{y_{2}^{\prime}-q_{2} y_{2}}{\lambda^{p-1}} \phi(\eta)-\frac{a_{2}}{\lambda^{p}} \phi(\eta)\left(y_{2}+\phi_{2}(\eta) y_{2}^{\beta_{2}}\right)\right\}=0 .
\end{aligned}
$$

Note that as $\eta \rightarrow+\infty$

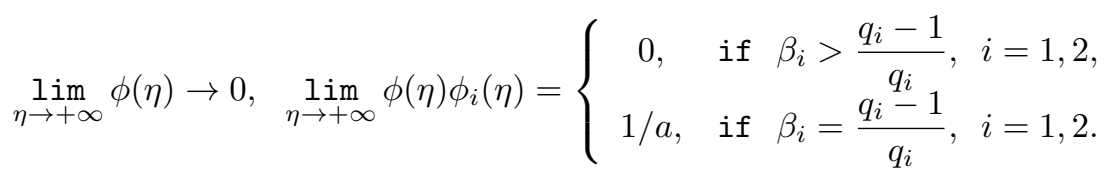

With into account the latest limits we will obtain from (17) the following system of algebraic equations:

$$
\begin{aligned}
& \left(y_{2}^{0}\right)^{m_{1}-1}\left(y_{1}^{0}\right)^{p-2}=c_{1}, \\
& \left(y_{1}^{0}\right)^{m_{2}-1}\left(y_{2}^{0}\right)^{p-2}=c_{2},
\end{aligned}
$$

if $\beta_{i}>\frac{q_{i}-1}{q_{i}}$ and

$$
\begin{aligned}
& q_{1}^{p-1}\left(y_{2}^{0}\right)^{m_{1}-1}\left(y_{1}^{0}\right)^{p-2}+\frac{a_{1}\left(y_{1}^{0}\right)^{\beta_{1}-1}}{a \lambda^{p} q_{1}}=\frac{1}{p \lambda^{p-1}} \\
& q_{2}^{p-1}\left(y_{1}^{0}\right)^{m_{2}-1}\left(y_{2}^{0}\right)^{p-2}+\frac{a_{2}\left(y_{2}^{0}\right)^{\beta_{2}-1}}{a \lambda^{p} q_{2}}=\frac{1}{p \lambda^{p-1}}
\end{aligned}
$$

if $\beta_{i}=\frac{q_{i}-1}{q_{i}}$.

Therefore we have the asymptotical representation (13).

In the case $\mathrm{p}=2$ or $\mathrm{m}=1$ in $(10)$, the properties of the different solutions as computing aspects of the system equation (10) were studied by many authors [12].

\section{Fast diffusion case $\left(q_{i}<0, i=1,2\right.$. $)$}

Consider now the case of fast diffusion. In this case we will study asymptotics of the regular solutions of self-similar system (10) as $\xi \rightarrow+\infty$ with the boundary condition

$$
\begin{aligned}
& f(0)=c_{1} \geqslant 0, f(\infty)=0, \\
& \psi(0)=c_{2} \geqslant 0, \psi(\infty)=0 .
\end{aligned}
$$

We will replace in $(10)$

$$
\begin{aligned}
& f(\xi)=\bar{f}(\xi) y_{1}(\eta), \\
& \psi(\xi)=\bar{\psi}(\xi) y_{2}(\eta),
\end{aligned}
$$

where $\eta=\ln \left(a+\xi^{\gamma}\right), \bar{f}=\left(a+\xi^{\gamma}\right)^{q_{1}}, \bar{\psi}=\left(a+\xi^{\gamma}\right)^{q_{2}}, a>0, \gamma, q_{1}, q_{2}$ are the numbers defined above.

Theorem 2. Let $q_{1}<0, q_{2}<0, \beta_{i}>1, i=1,2$. Then solutions of system (10) have the asymptotics

$$
\begin{aligned}
& f(\xi)=y_{1}^{0} \bar{f}(\xi)(1+o(1)), \\
& \psi(\xi)=y_{2}^{0} \bar{\psi}(\xi)(1+o(1))
\end{aligned}
$$


as $\eta \rightarrow \infty$ when one of the following conditions is satisfied:

$$
\begin{aligned}
& \text { I. }(N-l)\left[\left(m_{i}-1\right)\left(m_{3-i}-1\right)-(p-2)^{2}\right]-(p-n-l)\left(p-m_{i}-1\right)>0 \\
& \text { and } \\
& \qquad a_{i}>\frac{p-\left(m_{i}+1\right)}{\left(m_{i}-1\right)\left(m_{3-i}-1\right)-(p-2)^{2}}, \quad i=1,2,
\end{aligned}
$$

or

$$
\text { II. } \quad(N-l)\left[\left(m_{i}-1\right)\left(m_{3-i}-1\right)-(p-2)^{2}\right]-(p-n-l)\left(p-m_{i}-1\right)<0
$$

and

$$
a_{i}<\frac{p-\left(m_{i}+1\right)}{\left(m_{i}-1\right)\left(m_{3-i}-1\right)-(p-2)^{2}}, \quad i=1,2,
$$

numbers $y_{1}^{0}, y_{2}^{0}$ are the roots of the following nonlinear algebraic equations

$$
\begin{aligned}
& \left(s+\lambda q_{1}\right)\left(\left|q_{1} y_{1}^{0}\right|\right)^{p-2}\left(y_{2}^{0}\right)^{m_{1}-1}+\frac{1}{p \lambda^{p-2}}-\frac{a_{1}}{q_{1} \lambda^{p-1}}=0 \\
& \left(s+\lambda q_{2}\right)\left(\left|q_{2} y_{2}^{0}\right|\right)^{p-2}\left(y_{1}^{0}\right)^{m_{2}-1}+\frac{1}{p \lambda^{p-2}}-\frac{a_{2}}{q_{2} \lambda^{p-1}}=0 .
\end{aligned}
$$

Proof. After replacement (20) system (10) take the following form

$$
\begin{gathered}
\frac{d}{d \eta} L_{i}\left(y_{1}, y_{2}\right)+\left(\frac{s}{\lambda} \phi_{1}(\eta)+q_{i}\right) L_{i}\left(y_{1}, y_{2}\right)+\frac{1}{p \gamma^{p-1}} \phi_{1}(\eta)\left(\frac{d y_{i}}{d \eta}+q_{i} y_{i}\right)+ \\
+\frac{a_{i}}{\gamma^{p}} \phi_{1}(\eta)\left(y_{i}+\phi_{2}(\eta) y_{i}^{\beta_{i}}\right)=0
\end{gathered}
$$

where

$$
\begin{gathered}
L_{i}\left(y_{1}, y_{2}\right)=y_{3-i}^{m_{i}-1}\left|\frac{d y_{i}}{d \eta}+q_{i} y_{i}\right|^{p-2}\left(\frac{d y_{i}}{d \eta}+q_{i} y_{i}\right), \\
\phi_{1}(\eta)=\frac{e^{\eta}}{e^{\eta}-a}, \quad \phi_{2}(\eta)=e^{\eta q_{i}\left(\beta_{i}-1\right)} \phi_{1}(\eta), \quad i=1,2 .
\end{gathered}
$$

Such studying of solutions of a system (10) as $\eta \rightarrow+\infty$ is reduced to studying of those solutions of system (10), each of which in some neighborhood of $\infty$ satisfies to inequalities

$$
\frac{d y_{i}}{d \eta}+q_{i} y_{i} \neq 0, \quad y_{i}(\eta)>0, i=1,2
$$

Passing in (23) to the limit at $\eta \rightarrow+\infty$ we obtain necessary conditions and system of algebraic equations (22). Rest of a proving is similar to above mentioned manner.

4. Case $\beta_{1}=\beta_{2}=1$.

Now consider the Cauchy problem for system

$$
\begin{aligned}
& \frac{\partial(\rho(x) u)}{\partial t}=\operatorname{div}\left(|x|^{n} v^{m_{1}-1}|\nabla u|^{p-2} \nabla u\right)+\rho(x) \gamma_{1}(t) u \\
& \frac{\partial(\rho(x) v)}{\partial t}=\operatorname{div}\left(|x|^{n} u^{m_{2}-1}|\nabla v|^{p-2} \nabla v\right)+\rho(x) \gamma_{2}(t) v
\end{aligned}
$$

We will prove a condition of localization of the problem $(24),(2)$. 
Introduce the notation

$$
\begin{gathered}
\bar{u}(t)=\exp \int_{0}^{t} \gamma_{1}(s) d s, \bar{v}(t)=\exp \int_{0}^{t} \gamma_{2}(s) d s \\
\varphi(|x|)=\frac{1}{p_{1}}|r|^{p_{1}}, \quad p_{1}=\frac{p-(n+l)}{p}, \\
|x|=\sqrt{\sum_{i=1}^{N} x_{i}^{2},} \begin{array}{r}
\tau(t)=\int_{0}^{t} \bar{v}^{m_{1}-1}(\eta) \bar{u}^{p-2}(\eta) d \eta=\int_{0}^{t} \bar{u}^{m_{2}-1}(\eta) \bar{v}^{p-2}(\eta) d \eta \\
u_{+}(t, x)=\bar{u}(t) \bar{f}(\xi), \quad \xi=|x| /[\tau(t)]^{1 / p} \\
v_{+}(t, x)=\bar{v}(t) \bar{\psi}(\xi),
\end{array}
\end{gathered}
$$

where

$$
\bar{f}(\xi)=A\left(a-\xi^{\gamma}\right)_{+}^{q_{1}}, \bar{\psi}(\xi)=B\left(a-\xi^{\gamma}\right)_{+}^{q_{2}},
$$

$$
\gamma=\frac{p}{p-1}, \quad q_{1}=\frac{(p-1)\left(p-\left(m_{1}+1\right)\right),}{(p-2)^{2}-\left(m_{1}-1\right)\left(m_{2}-1\right)}, \quad q_{2}=\frac{(p-1)\left(p-\left(m_{2}+1\right)\right)}{(p-2)^{2}-\left(m_{1}-1\right)\left(m_{2}-1\right)},
$$

$a>0, A$ and $B$ are positive constants.

We notice that functions $u_{+}(t, x), v_{+}(t, x)$ are Zeldovich-Barenblatt type solutions to system (24). Here it constructed for the system (24) at the first.

It is hold the following

Theorem 3. Let $p>n+1, N>1, \exp \left(\int_{0}^{t} \gamma_{i}(\mu) d \mu\right)<+\infty, i=1,2, \tau(t)<+\infty$ for $\forall t>0$ and

$$
u_{0}(x) \leqslant u_{+}(0, x), v_{0}(x) \leqslant v_{+}(0, x), x \in \mathbb{R}^{N} .
$$

Then the solution of the system (1) is specially localized.

Proof. In order to prove Theorem 3 we use the following change of variables in system (1)

$$
\begin{aligned}
& u(t, x)=\bar{u}(t) w(\tau(t), \varphi(|x|)), \\
& v(t, x)=\bar{v}(t) z(\tau(t), \varphi(|x|)) .
\end{aligned}
$$

Then the system reduces to the form

$$
\begin{aligned}
& L_{3}(w, z)=-\frac{\partial w}{\partial \tau}+\varphi^{1-s} \frac{\partial}{\partial \varphi}\left(\varphi^{s-1} z^{m_{1}-1}\left|\frac{\partial w}{\partial \varphi}\right|^{p-2} \frac{\partial w}{\partial \varphi}\right)=0 \\
& L_{4}(w, z)=-\frac{\partial z}{\partial \tau}+\varphi^{1-s} \frac{\partial}{\partial \varphi}\left(\varphi^{s-1} w^{m_{2}-1}\left|\frac{\partial z}{\partial \varphi}\right|^{p-2} \frac{\partial z}{\partial \varphi}\right)=0
\end{aligned}
$$

where $s=p \frac{N-l}{p-(n+l)}, p>n+l$.

Set in $(26)$

$$
\begin{aligned}
& w(\tau, x)=f(\xi), \quad \xi=\frac{\varphi|x|}{\tau^{1 / p}} \\
& z(\tau, x)=\psi(\xi), \quad
\end{aligned}
$$

Then we have from (26) the self similar system equation

$$
\begin{aligned}
& L_{3}(f, \psi)=\xi^{1-s} \frac{d}{d \xi}\left(\xi^{s-1} \psi^{m_{1}-1}\left|\frac{d f}{d \xi}\right|^{p-2} \frac{d f}{d \xi}\right)+\frac{\xi}{p} \frac{d f}{d \xi}=0 \\
& L_{4}(f, \psi)=\xi^{1-s} \frac{d}{d \xi}\left(\xi^{s-1} f^{m_{2}-1}\left|\frac{d \psi}{d \xi}\right|^{p-2} \frac{d \psi}{d \xi}\right)+\frac{\xi}{p} \frac{d \psi}{d \xi}=0
\end{aligned}
$$


Notice the functions $\bar{f}(\xi), \bar{\psi}(\xi)$ have properties

$$
\begin{aligned}
& 0 \leqslant \bar{f}(\xi), \quad \xi^{s-1} \bar{\psi}^{m_{1}-1}\left|\frac{d \bar{f}}{d \xi}\right|^{p-2} \frac{d \bar{f}}{d \xi}=-A^{p-1} B^{m_{1}-1}\left(\lambda \gamma_{1}\right)^{p-1} \xi^{s} \bar{f}(\xi) \in C(0, \infty), \\
& 0 \leqslant \bar{\psi}(\xi), \quad \xi^{s-1} \bar{f}^{m_{2}-1}\left|\frac{d \bar{\psi}}{d \xi}\right|^{p-2} \frac{d \bar{\psi}}{d \xi}=-B^{p-1} A^{m_{2}-1}\left(\lambda \gamma_{2}\right)^{p-1} \xi^{s} \bar{\psi}(\xi) \in C(0, \infty) .
\end{aligned}
$$

To complete proof of the Theorem 3 it is sufficient to check conditions

$$
L_{3}(\bar{f}, \bar{\psi}) \leqslant 0, \quad L_{4}(\bar{f}, \bar{\psi}) \leqslant 0, \quad \text { in }|\xi|<a^{(p-1) / p}
$$

according to a comparison principle [11].

In fact, since

$$
\begin{gathered}
\xi^{N-1} \bar{\psi}^{m_{1}-1}\left|\frac{d \bar{f}}{d \bar{\xi}}\right|^{p-2} \frac{d \bar{f}}{d \xi}=-A^{p-1} B^{m_{1}-1}\left(\lambda \gamma_{1}\right)^{p-1} \xi^{N} \bar{f}(\xi), \\
\xi^{N-1} \bar{f}^{m_{2}-1}\left|\frac{d \bar{\psi}}{d \xi}\right|^{p-2} \frac{d \bar{\psi}}{d \xi}=-B^{p-1} A^{m_{2}-1}\left(\lambda \gamma_{2}\right)^{p-1} \xi^{N} \bar{\psi}(\xi), \\
\left(\gamma_{1}-1\right)(p-1)+\left(m_{1}-1\right) \gamma_{2}=\gamma_{1},\left(\left(m_{2}-1\right) \gamma_{1}+(p-1) \gamma_{2}=\gamma_{2},\right. \text { we have } \\
\xi^{1-N} \frac{d}{d \xi}\left(\xi^{N-1} \bar{\psi}^{m_{1}-1}\left|\frac{d \bar{f}}{d \bar{\xi}}\right|^{p-2} \frac{d \bar{f}}{d \xi}\right)=-A^{p-1} B^{m_{1}-1}\left(\lambda \gamma_{1}\right)^{p-1}\left(N+\xi \frac{d \bar{f}}{d \xi}\right) \\
\xi^{1-N} \frac{d}{d \xi}\left(\xi^{N-1} \bar{f}^{m_{2}-1}\left|\frac{d \bar{\psi}}{d \xi}\right|^{p-2} \frac{d \bar{\psi}}{d \xi}\right)=-B^{p-1} A^{m_{2}-1}\left(\lambda \gamma_{2}\right)^{p-1}\left(N+\xi \frac{d \bar{\psi}}{d \xi}\right) .
\end{gathered}
$$

Therefore

$$
L_{3}(\bar{f}, \bar{\psi})=-\frac{N}{p} \bar{f} \leqslant 0, \quad L_{4}(\bar{f}, \bar{\psi})=-\frac{N}{p} \bar{\psi} \leqslant 0, \quad i f|\xi|<a^{(p-1) / p}
$$

if numbers $A, B$ are the roots of the system of algebraic equations

$$
\begin{aligned}
& A^{p-1} B^{m_{1}-1}\left(\lambda \gamma_{1}\right)^{p-1}=1 / p, \\
& B^{p-1} A^{m_{2}-1}\left(\lambda \gamma_{2}\right)^{p-1}=1 / p .
\end{aligned}
$$

Hence

$$
L_{3}(f, \psi) \leqslant 0, \quad L_{4}(f, \psi) \leqslant 0,
$$

in $D=(t, x): t>0,|x|<l(t), l(t)=a^{(p-1) / p}[\tau(t)]^{1 /(p-l-n)}$.

Applying comparison principle [11] we have for solution of the problem (24), (2)

$$
u(t, x) \leqslant u_{+}(t, x), v(t, x) \leqslant v_{+}(t, x), \text { in } Q .
$$

We notice that the functions $w(\tau, x), z(\tau, x)$ have the properties

$$
w(\tau, x)=z(\tau, x) \equiv 0, \text { when }|x| \geqslant a^{(p-1) / p}[\tau(t)]^{1 /(p-l-n)} .
$$

In this case for the free boundary we have an estimate

$$
|x(t)| \leqslant a^{(p-1) / p}[\tau(t)]^{1 /(p-l-n)} \text { for } t>0 .
$$

Therefore if $\tau(t)<\infty$ for all $t>0$, then there place a space localization of a solution of the system (1) according condition of the theorem (3). Theorem 3 is proved.

We notice that in the case

$$
w(\tau, x)=z(\tau, x) \equiv 0, \text { when }|x| \geqslant a^{(p-1) / p}[\tau(t)]^{1 /(p-l-n)} .
$$

and $\tau(t) \rightarrow \infty$ when $t \rightarrow \infty$ there is a finite speed of perturbation. 


\section{Results of numerical experiments and visualization of solutions.}

For numerical solution of the problem the equation in two dimensions was used method Alternating Direction Implicit (ADI) in a combination to the method of balance. Iterative processes were constructed on the method, with Picard, Newton and a special method. In special linearization the members $u^{\beta_{1}}, v^{\beta_{2}}$, in system (1) are presented as $u_{k}^{\beta_{1}}=u_{k-1}^{\beta_{1}-1} u_{k}, v_{k}^{\beta_{2}}=v_{k-1}^{\beta_{2}-1} v_{k}$, where $u_{0}, v_{0}$, the solutions of the system of ordinary differential equation

$$
\frac{d u_{0}}{d t}=u_{0}^{\beta_{1}}, \frac{d v_{0}}{d t}=v_{0}^{\beta_{2}}
$$

Results of computational experiments show, that all listed iterative methods are effective for solving nonlinear problems and lead to nonlinear effects if we use as initial approximation the solutions of self-similar equations constructed by the method of nonlinear splitting and by the method of standard equation $[1,5,6]$. As it was expected, for obtaining some fixed accuracy the method of Newton requires smaller number of iterations, than the methods of Picard and the special method due to a good choice of an initial approximation. We observe that in each of the considered cases Newton's method has the best convergence due to the good initial approximation.

Below numerical results for one of solutions to problem (1)-(2) in the two-dimensional cases (Fig. 1). The results of the numerical experiment gives the effect of a finite speed of perturbation of a solution, and localization of a solution depending on the values of numerical parameters. The computational experiments were carried out for a slow and a quick diffusion cases.

\begin{tabular}{|l|r|r|}
\hline \multicolumn{1}{|c|}{ parameters } & $t=1$ & $t=20$ \\
\hline$m_{1}=0.2, m_{2}=0.2, p=2.8$ & & \\
$\beta_{1}=5, l_{1}=2$ & & \\
$\beta_{2}=7, l_{2}=3$ & & \\
$\beta_{1} \beta_{2}>1$ & & \\
$m_{i}+p-3=0$ & & \\
eps $=10^{-3}$ & & \\
& &
\end{tabular}

\begin{tabular}{|l|r|r|}
\hline$m_{1}=0.2, m_{2}=0.7, p=2.1$ & \\
$\beta_{1}=5, l_{1}=2$ & \\
$\beta_{2}=7, l_{2}=3$ & \\
$\beta_{1} \beta_{2}>1$ & \\
$m_{i}+p-3<0$ & & \\
eps $=10^{-3}$ & &
\end{tabular}




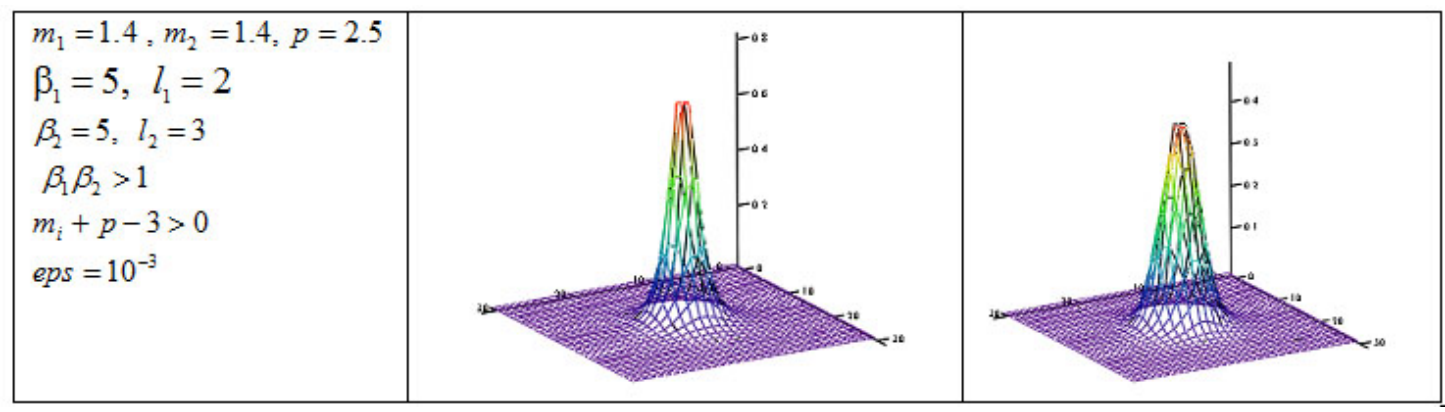

Fig. 1.

\section{References}

[1] M.Aripov, Sh.A.Sadullaeva. To solutions of one nondivergent type parabolic equation with double nonlinearity, Advances and Progress in Analysis, 2010, 1-18.

[2] M.Aripov, Sh.A Sadullaeva. To properties of the equation of reaction diffusion with double nonlinearity and distributed parameters, Journal of Sibirian Federal University. Mathematics \& Physics, 6(2013), 157-167.

[3] M.Aripov, Approximate self-similar approach for solving of quasilinear parabolic equation, Experimentation, Modeling and Computation in Flow, Turbulence and Combustion. Wiley, 2(1997), 19-26.

[4] M.Aripov. Asymptotic of the solution of the non-Newton polytropical filtration equation, Zeitschrift fur Angewandte Mathematik und Mechanik, 80(2000), no. 3, 767-768.

[5] M.Aripov, J.Muhammadiev. Asymptotic behavior of automodel solutions for one system of quasilinear equations of parabolic type, Buletin Stiintific-Universitatea din Pitesti, Seria Matematica i Informatica, 3(1999), 19-40.

[6] M.Aripov. Method of the standard equation for the solution of the nonlinear value problem, Tashkent, Fan, 1988.

[7] R.Kersner, J.Reyes, A.Tesei. One a class of nonlinear parabolic equations with variable density and absorption advances, Diff. Equations, 7(2002), 15-176.

[8] I.Kombe, Double nonlinear parabolic equations with singular lower order term, Nonlinear Analysis, 56(2004), 185-199.

[9] S.N.Dimova, M.S.Kastchiev, M.G.Koleva, D.P.Vasileva. Numerical analysis of the blow-up regimes of combustion of two-component nonlinear heat-conducting medium, Journal of applied mathematics and mathematical physics, 35(1995), no. 3, 303-319.

[10] B.H.Gilding, L.A.Pelletier. On a class of similarity of the porous media equation. 2, J. Math. Anal. and Appl., 57(1977), 52-538.

[11] A.A.Samarskii, V.A Galaktionov, S.P.Kurdyumov, A.P.Mikhajlov, Blow-up in quasilinear parabolic equations, Berlin, 4, Walter de Grueter, 1995.

[12] S.P.Kurdyumov, E.S.Kurkina, O.V.Telkovskaya, Regimes with sharpening in two-component media, Matem. Mod., 1(1989), no. 1, 34-50. 
[13] B.P.Demidovich. Lectures on mathematical theory of stability, Moscow, Nauka, 1967 (in Russian).

\section{Численное исследование решений системы реакции-диффузии с переменной плотностью}

Шахло А. Садуллаева

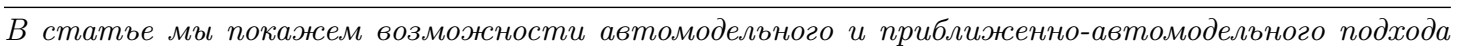
$\kappa$ исследованию взаимной системы реакции-диффузии нелинейных уравнений. Изучаются асимптотические поведения компактно распространенных решений и свободные границы. На основе полученных качественных свойств проведены численные расчеты. При этом решения представляются в визуальной форме по времени.

Ключевые слова: двойная нелинейность, система реакиии-диффузии, автомодельное уравнение, асимптотика решений, численное решение. 